\title{
Composite Technical Solutions in the Modern Innovation System of Project Development and Their Influence on the Process of Creating Complex Software Products as Part of These Projects
}

\author{
Konstantin A. Piliugin, Ph.D. \\ Voronezh, Russian Federation
}

\section{Influence of industrial fashion}

The current state of equipment and technology forces us to much more deeply link various scientific and technological aspects in the projects under development and compare their possible solutions with the provisions of TIPS (Theory for Inventive Problem Solving) and AIPS (Algorithm for Inventive Problem Solving).

To begin the analysis, denote the terminology:

- Technical system (TS)

- Main useful function (MUF) of the developed technical system

- Elementary useful function (UF) of the developed technical system

Main useful function (MUF) is the main object and purpose of development, of course, provided that it is defined correctly and its definition takes into account all possible functional loads that must be met by TS, especially when complex software products and their commercial functions and technological derivatives are included in its configuration.

For a set of useful functions performed by TS, one can always find a more general useful function directly reflecting the purpose of TS, the purpose of its existence and activities (and coinciding with them, especially due to the presence and necessity of introducing into the structure and basic functions of TS complex software products and, in turn - their derived elements, including components made and corresponding to the parameters of cybernetic systems, equivalent to artificial intelligence systems).

This general TS function is called the main useful MUF of the whole TS, in contrast to the elementary useful functions (hereinafter referred to as simply useful, UF), which together 
and separately ensure the implementation of MUF. The relationship between MUF and UF is the same as between the system and its subsystems. MUF refers to the system as a whole, and UF to its subsystems.

The positive effect, especially from complex TS, today largely depends on the degree to which complex software products are included in TS and their combinations with purely technological and constructive solutions, especially with complex active control systems and remote processor control systems, and operational information transfer to controlling computers.

Any change in TS that increases the capabilities of this TS in meeting the needs of super-systems (and society as well) is an improvement of the system.

The improvement in TS is manifested in the following changes in the system at the level of external functioning:

- quantitative growth of useful "capabilities" of TS - transformation of non-useful "capabilities" into useful ones; the use of complex software products to increase the quantitative and qualitative growth of the useful capabilities of the technical system;

- elimination of harmful "capabilities" until they turn into useful ones; the use of complex software products and their combinations and articulations with designer systems and functional blocks to increase the quantitative and qualitative growth of the useful capabilities of the technical system;

- an increase in the ratio of the useful output to the input, that is, an increase in the TS efficiency, including due to the original characteristics and complex functional features included in the technical system of complex software products.

\section{Availability and constant intensive development of software products}

Any technical system that is being developed today cannot function in a modern technological infrastructure and in the system of production and commercial interconnections that has developed in an innovative economy without corresponding software products and their derivatives, the technical characteristics of which must be functionally and systemically integrated into the technical system.

Since software solutions are by definition not technical solutions and cannot be autonomously represented as objects of inventions, including integrative ones, the introduction 
of software solutions of their combinations and products into technical systems, primarily for patent protection of such systems, requires strict and system linking of the elementary useful functions of each functionally complete element of such systems with the complex of functions included in the main useful function of the whole technical system.

\section{Change of rules and criteria of industrial design}

Globalization of the economy leads to the need for a much more comprehensive and

in-depth analysis of designer developments and functional characteristics of the products and technologies under development.

I propose to consider this thesis on the example of options for complex water treatment used for technological needs.

1. Treatment of liquids containing organic materials in combination with heavy metals

To separate a liquid into fractions with a high concentration of organic substances and into a liquid with heavy metal ions, a combined treatment is used, where the properties and parameters of the formed foam change when the pressure of compressed air changes.

A liquid with a high concentration of organic substances is separated with the foam, and after removing the foam, a liquid containing predominantly heavy metal ions remains.

But liquid treatment in the field requires other design solutions that meet the requirements of standards and are quite reliable. 


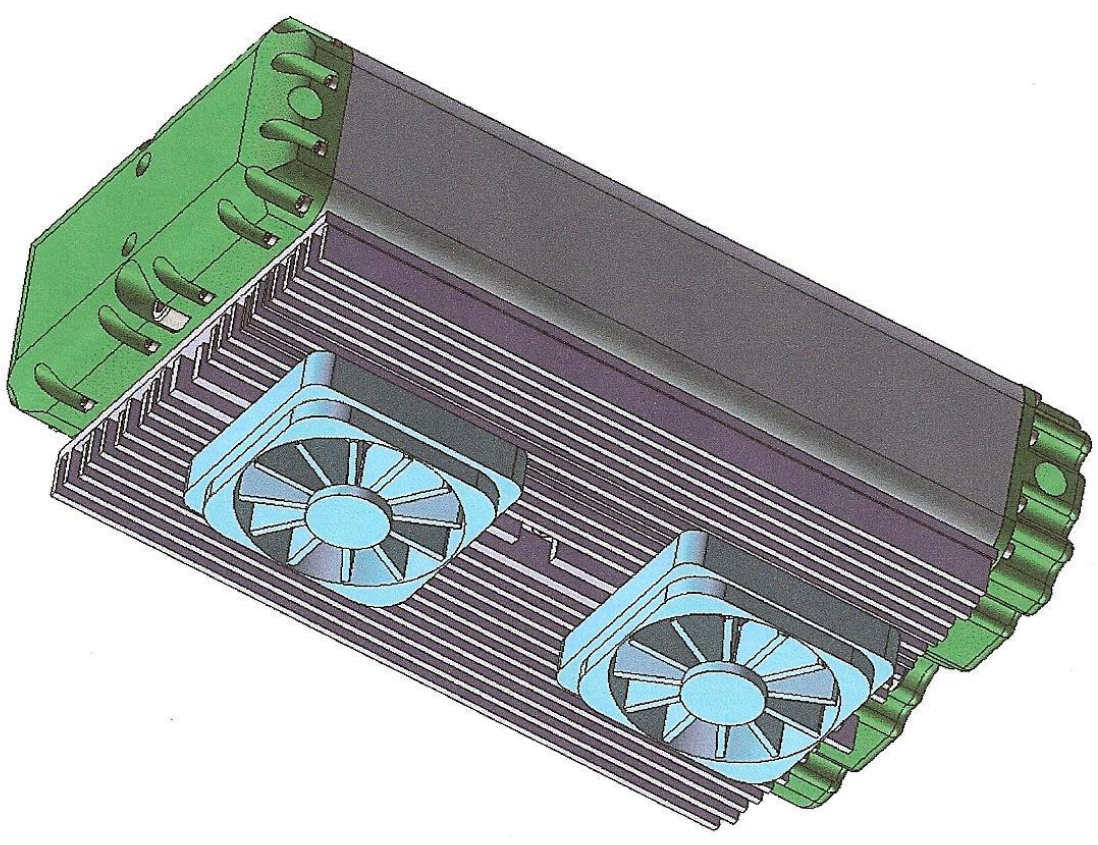

Figure 1. Model of an autonomous device for water regeneration in the field using natural materials - zeolite or other aluminosilicates.

Such a solution should be created on the basis of extremely simple design resources, at the same time ensuring the implementation of both elementary useful functions and full compliance with the Main Useful Function of the device. 


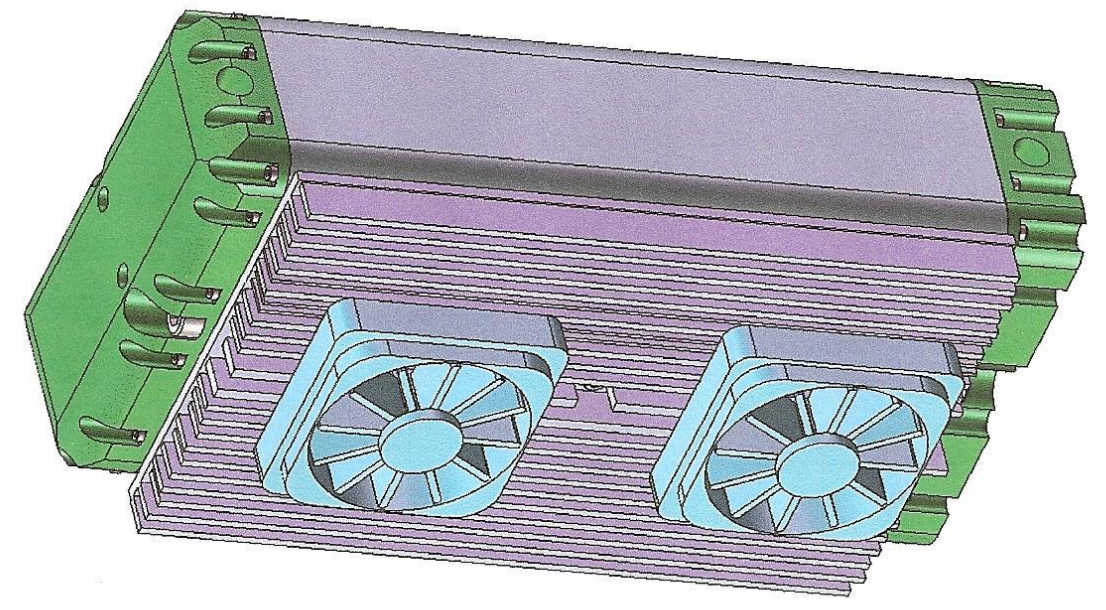

Figure 2. The model of an autonomous device for regeneration of radioactive water in the field using natural materials, such as crushed algae of the Ozol type, and also zeolite or other aluminosilicates combining with them, with the main useful function of absorption of radioactive ions of heavy metals, such as molybdenum isotopes, ruthenium, etc.

2. Treatment of liquids with acidic background, containing heavy metals in combination with organic acids.

Typically, these liquids contain complex contaminants (TSS, TDS, BOD, COD) and to extract heavy metals, it is necessary to separate the fractions of contaminants first.

To separate a liquid into fractions with a high concentration of organic substances and into a liquid with heavy metal ions, a combined treatment is used, where the properties and parameters of the formed foam change when the pressure of compressed air changes.

A liquid with a high concentration of organic substances is separated with the foam, and after removing the foam, a liquid containing predominantly heavy metal ions remains.

In addition, active homogeneous treatment with air leads to primary formation of oxides which simplifies the process of further treatment of water or an aqueous solution. 


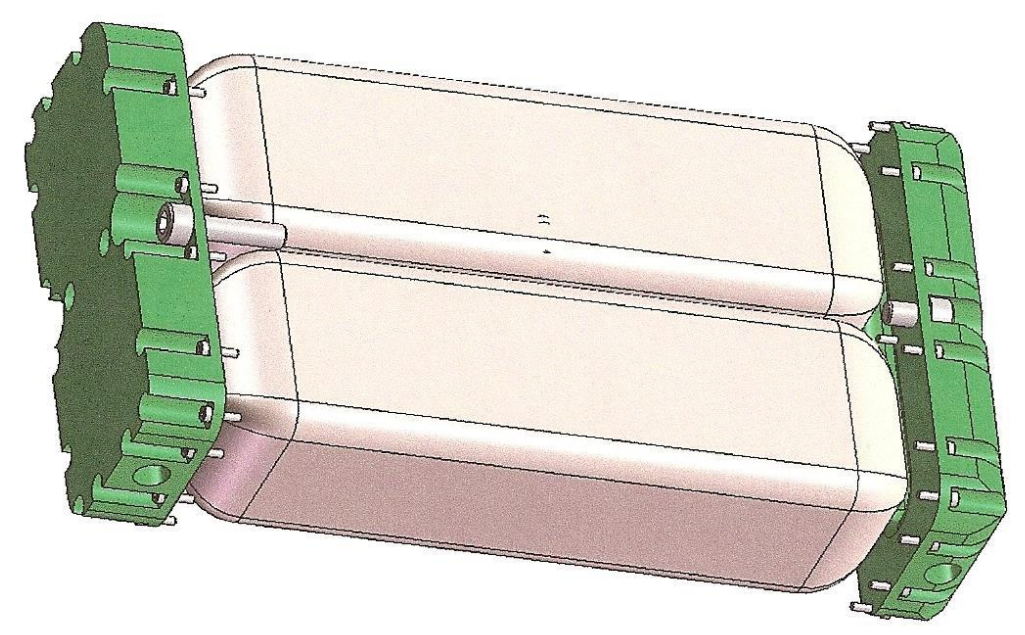

Figure 3. Models of liquid-permeable capsules with granulated active ion-exchange materials, mainly natural, such as, for example, zeolite, ozol, activated carbon, etc.

The same design of working capsules can be used for synthetic ion-exchange resins, and natural materials can be combined with synthetic ones.

As, for example, a mixture of zeolite granules with synthetic ion-exchange resin cationite, which allows to solve the problem of cooling water regeneration in atomic reactors.

The same mixture of zeolite granules can be used with ion-exchange resin - anionite. 

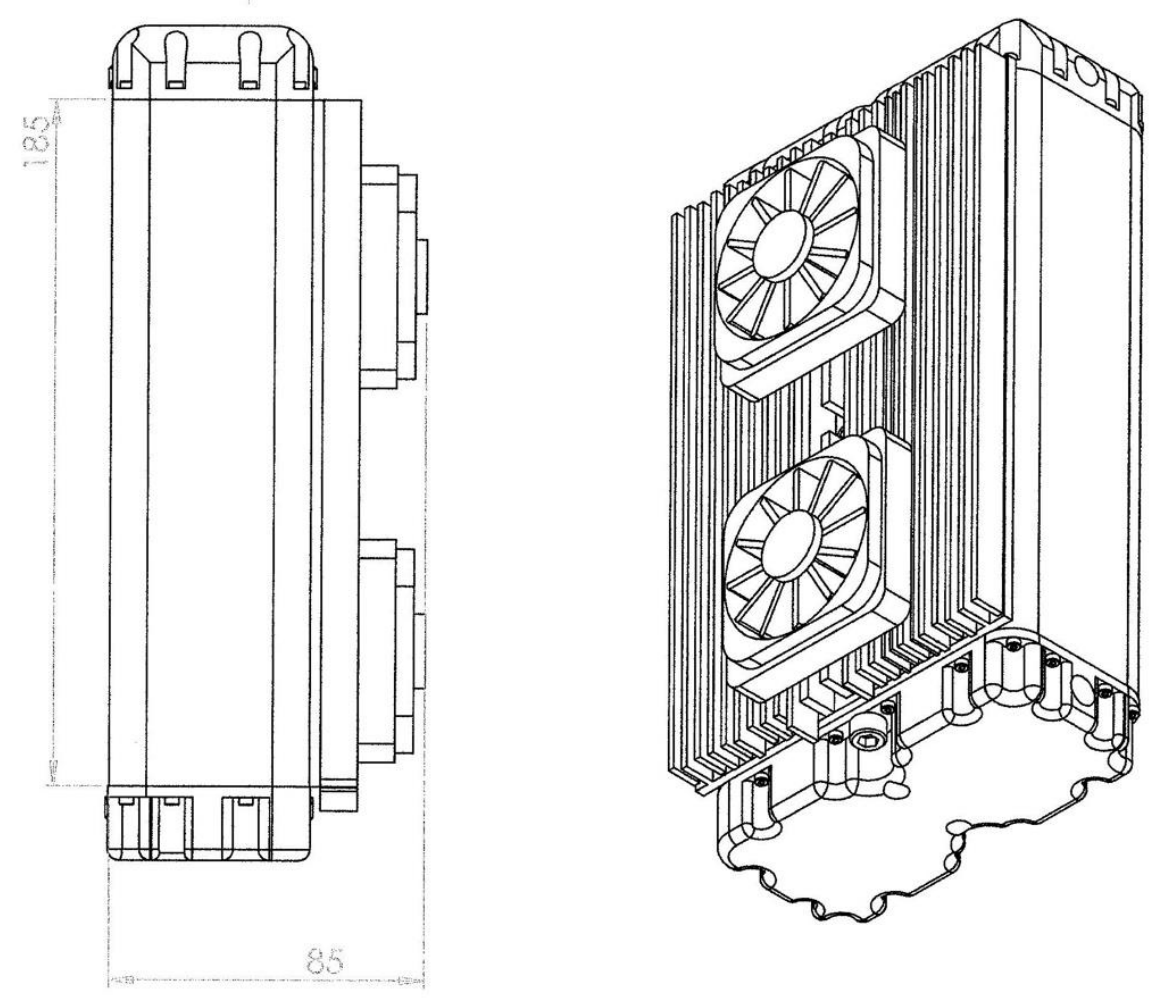

Figure 4. General view of the autonomous device for regeneration of aqueous process solutions; The device contains a number of composite technical solutions which can be controlled from the control processor with special software; As can be seen from the drawing, the device has extremely small dimensions.

3. Treatment of liquids with a high alkali content and a rich background of various contaminants, both organic and inorganic, containing heavy metals in combination with heavy metal salts.

Typically, these liquids contain complex contaminants (TSS, TDS, BOD, COD) and to extract heavy metals, it is necessary to separate the fractions of contaminants first.

To separate a liquid into fractions with a high concentration of organic substances and into a liquid with heavy metal ions, a combined treatment is used, where the properties and parameters of the formed foam change when the pressure of compressed air changes.

A liquid with a high concentration of organic substances is separated with the foam, and after removing the foam, a liquid containing predominantly heavy metal ions remains. 
4. Formation of a kind of vortex cutter for cleaning surfaces.

Formation of a vortex tube creates active cavitation on the surface being cleaned and washed, which increases the efficiency of cleaning and washing 3-5 times and reduces water consumption required for these technological operations 1.8-2.5 times.

5. Technological Pools for complex washing of large parts.

In this case, devices for foam formation (foam generators) are used to create a mode of uniform, active, and aggressive pulsation of a liquid with minimum size of gas bubbles and raising the level of liquid in the pool during the operation of foam generators, which makes it possible to reduce the volume of contaminated water during washing by $25-35 \%$.

6. Homogeneous saturation of process solutions and liquids with gas.

The use of foam generators or their structural equivalents increases the efficiency and reduces the cost of saturation of various liquids with various gases.

At the same time, the foam generator technology provides, in the process of saturation, complete control of the geometry of compressed gas bubbles in real time.

7. Rolled steel cooling in the metallurgical industry.

Water, or an aqueous solution can be modified by introducing a stream of bubbles of compressed gas (for example nitrogen), with sizes of no more than 25-50 microns.

When a gas is introduced into a liquid, it lowers its temperature, and further, when it comes into contact with the cooled surface and when the shells of air bubbles break, gas expansion and more substantial cooling occur.

This reduces energy costs for cooling $2-3$ times and significantly increases the cooling process efficiency.

The cooling process can be carried out at the same energy consumption in parallel with the process of washing or cleaning the cooled surfaces.

8. Technologies and applied technique for activating classical flotation processes. 
Devices for foam formation (foam generators) can be effectively used to produce uniformly sized air bubbles.

At the same time, the adjustment of technological modes is considerably simplified, since it is carried out only by adjusting the parameters of compressed air, and the bubble diameters are determined only by the foam generator geometry.

This significantly reduces energy consumption and improves the cleaning quality.

9. Combined flotation technologies with dynamic foaming

Foam generators can be used to create a combined flotation process, where foam is formed at the first stage, at high air pressure (6-8 bar), and after its separation, at low pressure ( $0.5-1.5$ bar), treatment is carried out by forming a uniform flow of air bubbles.

10. Technologies of forced separation of liquids and contaminants when low concentrations of detergents are introduced into the treated fluid.

In many cases, the liquid to be treated does not contain foaming substances.

In this case, it is advisable to introduce a small amount of detergent into the liquid (no more than 25 milligrams per liter of the liquid being treated).

The active foam formed during the foam generator operation makes it possible to significantly reduce the concentration of contaminants before transition of the foam generator to production of air bubbles and, accordingly, transition to the classical flotation mode.

11. Technologies of dynamic mixing of liquids of various properties

When a liquid is supplied to the foam generator instead of air, and a flow discharged from the foam generator enters another liquid, an effective homogeneous dynamic mixing process takes place, which is 5 to 7 times more effective than mechanical mixing.

12. Water saving technologies for process baths.

The use of foam generators in various process baths, the effect of raising the level of water or any other liquid in the bath, lowers the level of water or any other technological solution in the bath by $25-30 \%$. 
This saves $10-15 \%$ of technological solutions, with low consumption of energy for the preparation of compressed air.

13. Technologies of formation of fine-dispersed and aerosol mist.

Above the surface of the vortex tube formed during the foam generator operation, a mist appears, consisting of air bubbles in the shell of liquid in which the foam generator operates.

For fine and precise washing technologies, it is preferable to apply a mist treatment with a high cavitation effect, which occurs when the air bubble shells break.

Effective mist formation requires air pressure of 7 to 8 bar at a flow rate determined on the basis of the volume of the liquid being treated.

14. Treatment of aqueous solutions with high concentration of contaminants.

Provided for pre-treatment of aqueous solutions before electrochemical or any other treatment.

For treatment, the aqueous solution foams and contaminants concentrate in the foam.

The more contaminants tend to foam, the more effective the treatment process.

Contaminants are removed with foam.

Effective foam formation requires air pressure within 6 to 8 bar, a flow rate is determined on the basis of the volume of the liquid being treated.

15. Processing of aqueous solutions containing chemical complexes.

Provided for pre-treatment of aqueous solutions before electrochemical or any other treatment.

In this case, foaming of an aqueous solution is used, where the solution with all impurities is oxidized, and all impurities are present in the foam composition, since water does not foam.

If the foam is permanently removed, the system comes to a point when all the substances that have foam formation properties are removed from the solution.

Effective foam formation, according to the experience, requires air pressure within 6 8 bar (depending on the process intensity, the flow rate is determined by experiments). 
The above 15 variants of the use of foam generators in technologies for regeneration of water and aqueous solutions show flexibility in the compositional formation of processes and give an idea of the potential for introducing software products into process equipment and process flowcharts of compositional technical solutions.

\section{A new look at the durability and quality of a new product, including containing} complex software products and their derivatives in the technical system

Dialectical contradiction in the technical system is aggravated in case of introducing into functions and parameters of its technical characteristics of a complex software product and its derivatives.

The source of development of conventional TS and TS modified with the introduction of a software product, as well as any object of the material world, is the law of unity and struggle of opposites - the universal law of development of nature, society, and technology.

Opposites are the sides of an object that are in mutually exclusive relationships. In this case, the side of an object or phenomenon is understood as everything that is somehow inherent in the object or phenomenon, characterizes it and can be comprehended.

Opposites in TS are "input" and "output", useful functions, costs, and "capabilities".

The interaction of opposites, when they both mutually presume and at the same time deny and exclude each other, is a dialectical contradiction.

Technical contradiction (TC) in the technical system is a dialectical contradiction, which manifests itself in the technical system as a deterioration of one side of TS at the level of external functioning (from the standpoint of super-system needs) while improving the other side of TS.

In other words, TC can be defined as the dialectical unity of interdependent positive and undesirable effects in TS.

In this situation, a complex software product, with proper and comprehensive consideration of all possible technical contradictions, should play a positive role and to a large extent should reduce the severity and influence of these contradictions on the main useful function of the technical system.

TC is always associated with some component of TS (element, group of elements, or interaction of elements), which is commonly called the nodal component (NC). 
This TS component is immediately related to two sides of TS, and a quantitative change in some parameter (or state) of this component leads to an improvement in one and a deterioration in the other side of TS.

Therefore, more precisely, TC should be defined as the dialectical unity of positive and undesirable effects interdependent on quantitative or qualitative changes in the nodal component of TS.

Physical contradiction. Technical contradiction in its form appears in TS at the level of its external functioning. At the level of internal functioning, no mutually exclusive relations between the parties to the system are observed: from a physical point of view, TS is in some one state determined by the laws of nature.

But if we set the task of eliminating TC within this TS, asserting positive and negating undesirable effects, then mutually exclusive relations will manifest at the level of internal functioning, in the form of incompatible requirements for the parameter (state) of the nodal component of TS, more precisely, to the physical state of NC.

Such contradictions are called physical (FC). FC manifests itself in setting of the problem of eliminating $\mathrm{TC}$, in other words, $\mathrm{FC}$ is a form of expression of the problem of

eliminating TC within this TS. FC resolution is to establish new forms of organization and movement of matter in TS, when both incompatible requirements for the NC state are met, or, according to specialists introducing software products into the technical system, to establish such a "form of movement where this contradiction is both realized and permitted."

This phenomenon comprises the need to introduce complex and integrated software products into modern innovative technical systems.

\section{References}

1. US patent application No, 11.10.2018. PROGRAMMING MODEL OF NEURAL NETWORK-ORIENTED HETEROGENEOUS COMPUTING PLATFORM / SUN; Xiaoming; et al.

2. US patent application No, 12.07.2018. POROUS NANO STRUCTURE USEFUL AS ENERGY STORAGE MATERIAL, AND METHOD OF MANUFACTURING SAME / OH; Il-Kwon; et al.

3. US patent application No, 26.07.2018. WATER-BASED DRILLING FLUID FOR PROTECTING FRACTURED RESERVOIRS, AND PREPARATION METHOD AND USE THEREOF / SUN; Jinsheng; et al. 
4. US patent application No, 30.08.2018. Laser frequency control and sensing system / Dinovitser; Alex.

5. US patent application No, 26.07.2018. A DEVICE, PROCESS, AND CATALYST INTENDED FOR DESULFURIZATION AND DEMERCAPTANIZATION OF GASEOUS HYDROCARBONSD, TYURINA; Liudmila Aleksandrovna; et al.

6. US patent application No, 09.08.2018. DATA AND TASK REALLOCATION IN DISTRIBUTED COMPUTING PLATFORMS / Liu; Liang; et al.

7. US patent application No, 26.07.2018. Computing System and Process for Digital Video Data Management and Scheduling / Carter; Colin Maxwell

8. US patent application No, 04.10.2018. SOFTWARE INSTALLATION ASSISTANCE METHOD AND SYSTEM / Chakra; Al; et al.

9. US patent application No, 26.07.2018. COMPOSITE MATERIAL FOR A SLIDING BEARING / Buerkle; Gunter; et al. 